\title{
Computational identification of multi-omic correlates of anticancer therapeutic response
}

\author{
Lindsay C Stetson ${ }^{1,2}$, Taylor Pearl ${ }^{3}$, Yanwen Chen ${ }^{1}$, Jill S Barnholtz-Sloan ${ }^{1,2^{*}}$ \\ From 9th International Conference of the Brazilian Association for Bioinformatics and Computational Biology \\ (X-Meeting 2013) \\ Recife, Brazil. 3-6 November 2013
}

\begin{abstract}
Background: A challenge in precision medicine is the transformation of genomic data into knowledge that can be used to stratify patients into treatment groups based on predicted clinical response. Although clinical trials remain the only way to truly measure drug toxicities and effectiveness, as a scientific community we lack the resources to clinically assess all drugs presently under development. Therefore, an effective preclinical model system that enables prediction of anticancer drug response could significantly speed the broader adoption of personalized medicine.
\end{abstract}

Results: Three large-scale pharmacogenomic studies have screened anticancer compounds in greater than 1000 distinct human cancer cell lines. We combined these datasets to generate and validate multi-omic predictors of drug response. We compared drug response signatures built using a penalized linear regression model and two non-linear machine learning techniques, random forest and support vector machine. The precision and robustness of each drug response signature was assessed using cross-validation across three independent datasets. Fifteen drugs were common among the datasets. We validated prediction signatures for eleven out of fifteen tested drugs (17-AAG, AZD0530, AZD6244, Erlotinib, Lapatinib, Nultin-3, Paclitaxel, PD0325901, PD0332991, PF02341066, and PLX4720).

Conclusions: Multi-omic predictors of drug response can be generated and validated for many drugs. Specifically, the random forest algorithm generated more precise and robust prediction signatures when compared to support vector machines and the more commonly used elastic net regression. The resulting drug response signatures can be used to stratify patients into treatment groups based on their individual tumor biology, with two major benefits: speeding the process of bringing preclinical drugs to market, and the repurposing and repositioning of existing anticancer therapies.

\section{Background}

A major challenge in precision medicine is the transformation of multi-omic data into knowledge that enables stratification of patients into treatment groups based on predicted clinical response. Some progress has been made to associate genetic lesions and expression profiles with drug response. The link between a patient's therapeutic response and somatic alterations in the cancer genome was established by the National Cancer Institute (NCI) using the NCI60 human tumor cell line anticancer drug screen [1]. The analysis done by the NCI led to the discovery that mutations in $B R A F$ and EGFR are highly

\footnotetext{
*Correspondence: jsb42@case.edu

${ }^{1}$ Case Comprehensive Cancer Center

Full list of author information is available at the end of the article
}

predictive of clinical response to kinase inhibitors [2,3]. Recently, the use of imatinib to selectively target the protein product of the $B C R-A B L$ translocation revolutionized treatment of chronic myeloid leukemia [4]. Nevertheless, many cancer drugs have yet to be linked to the biomarkers necessary for assessing the effectiveness of the proposed therapeutic intervention.

Using multi-omic data to develop a statistical model predictive of drug response is not a trivial task. Single gene alterations discovered by linear regression techniques are often false-positive discoveries that mask the underlying biological pathway dysregulation driving drug response. There remains an urgent need to use multivariate and non-linear statistical methods to build robust multi-omic 
predictors of drug response that incorporate information from a myriad of biological alterations.

Although clinical trials remain the only way to truly measure drug toxicities and effectiveness, as a scientific community we lack the resources to clinically assess all drugs presently under development. Therefore, there is great enthusiasm to develop a preclinical system that would allow for high-throughput testing of cancer cell lines against large numbers of drug compounds in parallel. Preclinical computational models predictive of the drug response could be built based on genomic and drug screening results. Drug response signatures could be confirmed using independent validation datasets and patient tumor samples. We acknowledge that biological findings in cell lines and animal model systems have not always validated in human tumors. However, successfully validated drug response signatures have the potential to significantly speed the personalized matching of drugs to patient based on the patient's unique tumor biology.

In March 2012, the results of two large-scale pharmacogenomic human cancer cell line screens were published in Nature [5,6]. The Cancer Cell Line Encyclopedia (CCLE), published by researchers at the Broad Institute, and the Cancer Genome Project (CGP), presented by scientists at the Sanger Institute, complement the existing NCI60 pharmacogenomic database. Analyzing these databases in tandem potentiates the discovery of powerful, independently validated biomarkers of drug response. In this study, we used the NCI60, CCLE, and CGP pharmacogenomic datasets and evaluated the effectiveness of different computational approaches in deriving multi-omic signatures predictive of drug response. To our knowledge, this is the first time that all three datasets have been analyzed in a single study. A previous study attempted to develop genomic predictors of drug response using only gene expression data from the CCLE and CGP datasets [7]. Here we present an integrative analysis of high-throughput genomic and transcriptomic data; the resulting multi-omic signatures of therapeutic drug response have been validated across independent datasets. Using non-linear machine learning techniques, we generated robust multiomic signatures that predict cellular response to 17-AAG, AZD0530, AZD6244, Erlotinib, Lapatinib, Nultin-3, Paclitaxel, PD0325901, PD0332991, PF02341066, and PLX4720.

\section{Materials}

To develop multi-omic predictors of anticancer therapeutic response we curated data from the CCLE, CGP, and NCI60 databases. The resulting datasets consisted of the gene expression (Affymetrix U133A and Affymetrix U133A plus 2.0), copy number variation (Affymetrix SNP6.0), and mutational status (targeted and whole exome sequencing) of 1299 distinct human cancer cell lines representing 35 cancer types. Our curated data also included the sensitivity of the cell lines to treatment with fifteen drugs common across the CGP and CCLE databases (see Table 1). This publicly available data can be downloaded at http://broadinstitute.org/ccle (CCLE), http://cancerrxgene.org (CGP), and http://discover.nci.nih. gov/cellminer/ (NCI60).

\section{Drug sensitivity}

Cellular drug sensitivity was measured as the concentration of drug, in micromolar units $(\mu \mathrm{M})$, needed to inhibit $50 \%$ of cellular growth $\left(\mathrm{IC}_{50}\right)$. The drug compounds were robotically added to cell cultures and after 72 hours cell viability was assessed by measuring the ATP content of the assay [further details can be found in the supplementary methods of 5,6$]$. In our analysis, models were trained using the $10 \%$ most sensitive and resistant cell lines (lowest and highest $\mathrm{IC}_{50}$ ) to each drug of interest. The utilization of the cell lines at the limits of drug response increased the likelihood of identifying the multi-omic features that drive drug response.

\section{Gene expression}

Raw gene expression CEL files were normalized using fRMA [8] and R Bioconductor [9] chip packages ('hthgu133a' for CGP and NCI60; 'hgu133plus' for CCLE). Probesets not common across the datasets were discarded. Batch effects within and between the CCLE, CGP, and NCI60 datasets were removed using the ComBat function from the R 'sva' package [10]. Finally, for each Entrez gene ID the R package 'jetset' [11] was used to select the best probeset for each gene such that each gene is represented by one probe (12,151 genes).

\section{Copy number variation}

Copy number segments for 426 cancer genes were predicted using the PICNIC algorithm [12]. The raw CNV values were converted into five categories: amplification (copy number of eight or more), partial amplification (copy number between three and seven), normal (copy number of two), partial deletion (copy number of one), and full deletion (copy number of zero).

\section{Mutational status}

The mutational status of sixty-four commonly mutated cancer genes was assessed. A gene was defined as mutated if a coding sequence variant was present. Additionally, for cell lines in the CGP and CCLE databases the presence or absence of commonly rearranged cancer genes was determined.

\section{Methods}

The approach used to build multi-omic signatures predictive of drug response is illustrated in Figure 1. The drug response signatures were generated using a two-step 
Table 1 Common drugs in the Cancer Genome Project (CGP) and Cancer Cell Line Encyclopedia (CCLE) datasets

\begin{tabular}{llll}
\hline Compound & Target(s) & Class & Organization \\
\hline 17-AAG* & HSP90 & Targeted other & Bristol-Myers Squibb \\
AZD0530 & SrC, Abl, EGFR & Kinase Inhibitor & AstraZeneca \\
AZD6244* & MEK & Kinase Inhibitor & AstraZeneca \\
Crizotinib* $_{\text {Erlotinib* }}^{\text {C-MET, ALK }}$ & EGFR & Kinase Inhibitor & Pfizer \\
Lapatinib* $_{\text {Nilotinib* }}^{\text {EGFR, HER2 }}$ & Abl/Bcr-Abl & Kinase Inhibitor & Genentech \\
Nutlin-3* & MDM2 & Kinase Inhibitor & GlaxoSmithKline \\
NVP-TAE684* & ALK & Kinase Inhibitor & Novartis \\
Paclitaxel* & Beta-Tubulin & Targeted other & Roche \\
PD-0325901 & MEK & Kinase Inhibitor & Novartis \\
PD-0332991 & CDK4/6 & Cytotoxic & Bristol-Myers Squibb \\
PHA-665752 & C-MET & Kinase Inhibitor & Pfizer \\
PLX4720 & RAF & Kinase Inhibitor & Pfizer \\
Sorafenib* & FLT3, C-KIT, PDGFR, RET, Raf kinases, VEGFR, KDR, FLT4 & Kinase Inhibitor & Pfizer \\
\hline
\end{tabular}

* Indicates compound found in CGP, CCLE and NCI60 datasets

procedure consisting of statistical feature selection, to reduce the complexity of the datasets, followed by a classification algorithm, to weight each feature's contribution to the overall prediction. The predictive models were generated using CGP data as input and subjected to ten-fold cross-validation with ten repetitions per fold. The outputs of the process were weighted multi-omic drug response signatures. A signature size of thirty has previously been reported as an optimal balance between clinical relevance

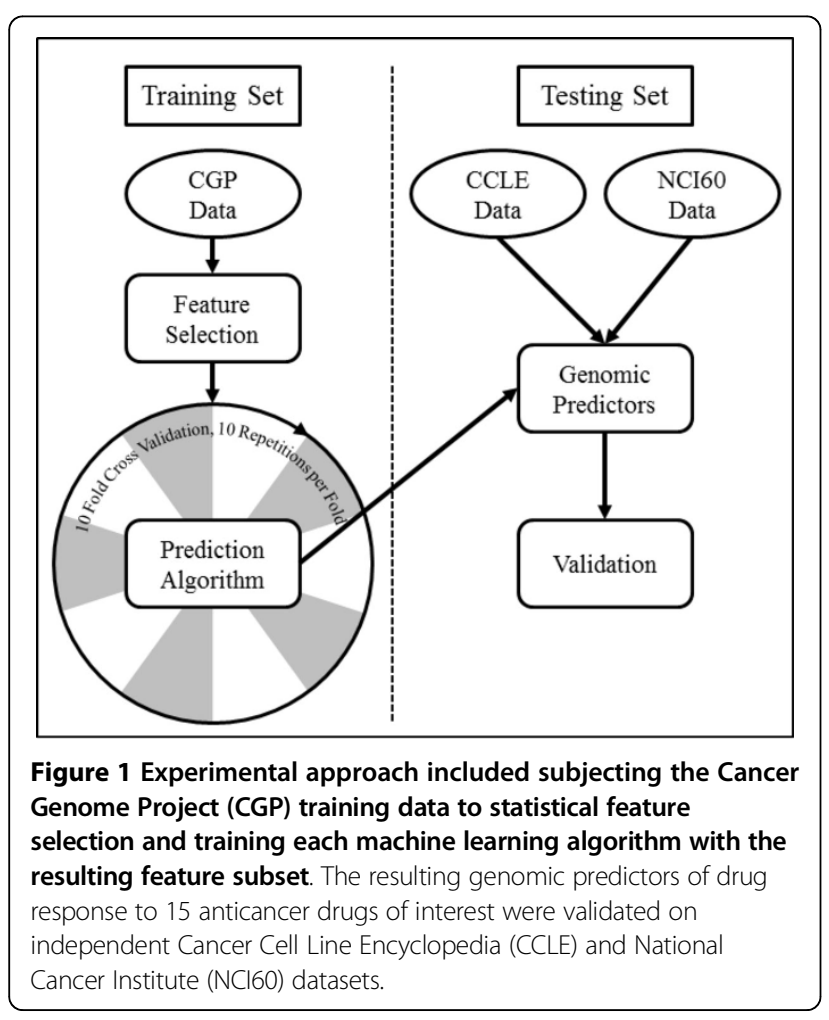

and genomic complexity $[13,14]$, therefore we limited our final predictive signatures to the top thirty features. The final signatures were then tested for accuracy and overfitting using the independent CCLE and NCI60 datasets.

\section{Feature selection}

The feature selection inputs were as follows:

1. A matrix of features $X \in P^{N, p}$, where $N$ was the total number of cell lines in the CGP dataset and $p$ was the number of multi-omic features (gene expression, $\mathrm{CNV}$, and mutation).

2. A vector of drug sensitivities, $Y \in P^{N, 1}$, where $\mathrm{N}$ was the number of cell lines treated with the drug of interest and the vector values were the corresponding cellular drug sensitivities (refer to section "Materials: Drug sensitivity").

For each drug the Wilcoxon Sum Rank Test (for continuous variables) was used to select genes whose expression was significantly differentially expressed between the $10 \%$ most sensitive and resistant cell lines (lowest and highest $\mathrm{IC}_{50}$ ). The Fisher's Exact Test (for categorical variables) was used for each drug to select genes whose mutational status and/or CNVs significantly differed between sensitive and resistant cell lines. The resulting machine learning input sets for each drug were comprised of the 1000 most differentially expressed features (as determined by Wilcoxon and Fischer's tests). The feature selection was completed using a custom script implemented in SAS.

\section{Machine learning prediction}

We assessed the performance of two machine learning methods, random forest and support vector machine 
(SVM), in generating accurate and precise multi-omic signatures predictive of drug response using the input sets for each drug comprised of the 1000 most differentially expressed features. We compared the performance of these models to the accuracy and precision of the drug response signature generated using elastic net regression, a type of penalized linear regression.

The random forest model was implemented in $\mathrm{R}$ using the 'RandomForestSRC' package $[15,16]$. We evaluated several combinations of forest size (ntree), number of features selected at each node (mtry), and node size (nodesize). The parameters resulting in the highest prediction accuracy were $n t r e e=2000, m t r y=$ feature set size $/ 3$, and nodesize $=1$. For each tree, the prediction error on the out-of-bag data was recorded. For each feature $x$ the out-of-bag cases were randomly permuted and the prediction error was recorded. The variable of importance for each feature was defined as the difference between the perturbed and unperturbed error rate averaged over all trees.

A support vector machine was implemented using the 'libsvm' R package [17]. The SVM was used was a type $C$ classification machine using a radial basis kernel. Features were ranked based on their weight magnitude $\left(\omega^{2}\right)$-a measure of class predictive ability.

The 'glmnet' $R$ package was used to implement elastic net regression [18]. We selected the same optimal regularization parameters used in the primary CGP and CCLE publications: $\alpha \varepsilon(0.2,1.0)$ and $\lambda=\mathrm{e}^{\gamma}$, where $\gamma \varepsilon$ $(-6,5)$-which minimized the root mean squared error using ten-fold cross-validation [5-7].

\section{Results}

We have evaluated the effectiveness of three computational approaches for deriving clinically relevant $m u l t i-$ omic signatures predictive of drug response. In our study, we compared elastic net regression, a commonly used linear method; support vector machine; and random forest, an effective ensemble method.

Signatures consisting of thirty multi-omic features were generated using the CGP pharmacogenomic database as a training set. The performance of these signatures in predicting drug response was assessed as precision. Precision was calculated as the ratio of true classifications of cellular drug sensitivity to all positive classification results. As illustrated in Figure 2, we observed significant and clinically relevant signature performance, precision greater than 0.80 , for twelve out of fifteen drugs. The random forest approach yielded the most precise results for ten out of the twelve prediction signatures generated (17-AAG, AZD6244, Erlotinib, Lapatinib, Nultin-3, Paclitaxel, PD0325901, PD0332991, PLX4720, Sorafenib). The support vector machine approach yielded the most precise results for two out of the twelve prediction signatures

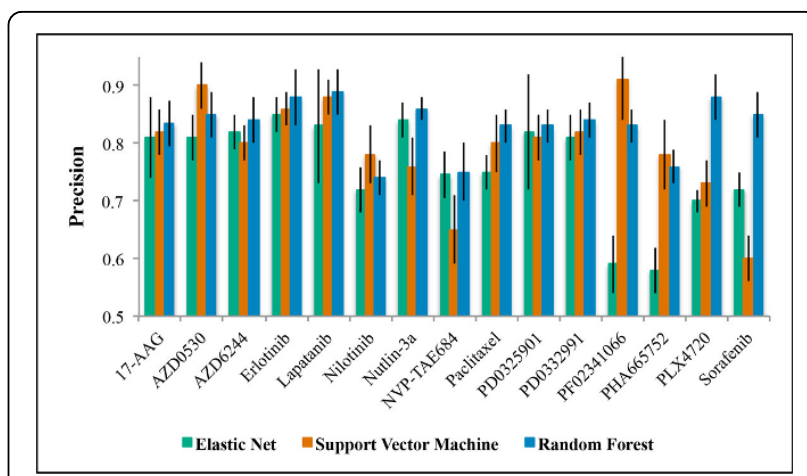

Figure 2 Mean prediction performance of multi-omic drug response signatures generated using elastic net regression, a support vector machine, or random forest on the Cancer Genome Project (CGP) data. Prediction performances (precision) are quantified as the proportion of true positive drug sensitive classifications to all positive classifications. Error bars represent the standard deviation of the precisions calculated during ten repetitions of ten-fold cross validation.

generated (AZD0530 and PF02341066). Elastic net regression failed to yield a top performing prediction signature for any of the fifteen drugs evaluated.

Independent validation of our generated signatures was performed using the CCLE and NCI60 datasets. As illustrated in Figure 3, eleven of the twelve drug response signatures developed using the CGP dataset successfully predicted, with a precision greater than 0.80 , drug response in the CCLE dataset (17-AAG, AZD0530, AZD6244, Erlotinib, Lapatinib, Nultin-3, Paclitaxel, PD0325901, PD0332991, PF02341066, and PLX4720). Using the NCI60 dataset we were able to predict drug response, with a precision greater than 0.80 , for seven out of eight signatures for drugs that are common across the CGP and NCI60 databases (17-AAG, AZD6244, Erlotinib, Lapatinib, Nultin-3, Paclitaxel, and PF02341066).

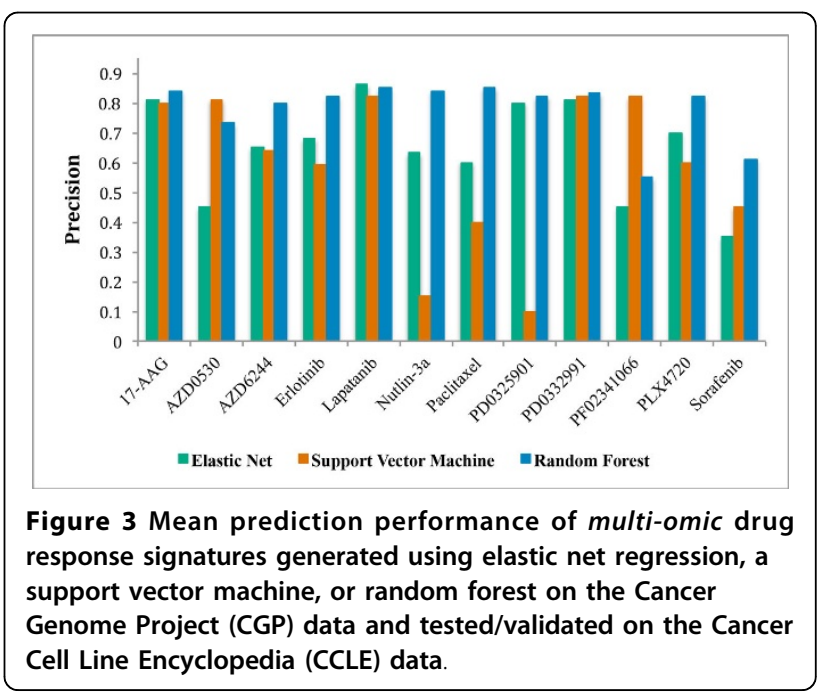




\section{Discussion}

Significant computational effort has been expended in the past ten years to build robust and clinically relevant predictors of drug response. Preclinical efforts have previously been limited by the lack of publically available data from which to build prediction sets. The publication of the CGP and CCLE pharmacogenomics datasets in March of 2012 made large-scale integrated analysis of multi-omic and drug response data possible [5,6]. To our knowledge, the genomic, transcriptomic, and drug profiling data contained in the CGP, CCLE, and NCI60 databases has not previously been analyzed in concert. We have combined these three datasets to generate and independently validate genomic correlates of anticancer drug response.

The purpose of our study was twofold: to show that precise and robust predictors of drug response could be built and to explore the use of multivariate linear and non-linear statistical methods in building the predictors. Previous studies have relied heavily upon commonly used penalized linear regression models in developing predictive genomic signatures, likely because of the challenges inherent to machine learning techniques[5-7,19]. While machine learning algorithms such as random forest and support vector machine can be more computationally intense and difficult to interpret, our work shows that the signatures derived there from have superior prediction power and robustness [15,20-23]. Non-linear machine learning algorithms are more effective in generating prediction signatures, as opposed to linear regression, because they make no distributional assumptions about the predictor variables and allow all features, including those with individually weak effects, to contribute to the model fit.

We generated multi-omic predictors of drug response to fifteen drugs of interest. During the signature-generation phase we created and validated predictive signatures using the CGP dataset (Figure 2). Using elastic net regression, eight of the fifteen signatures successfully predicted drug response with a precision greater than 0.80 (17-AAG, AZD0530, AZD6244, Erlotinib, Lapatinib, Nultin-3, PD0325901, PD0332991). Using a support vector machine, nine of the fifteen signatures successfully predicted drug response with a precision greater than 0.80 (17-AAG, AZD0530, AZD6244, Erlotinib, Lapatinib, Paclitaxel, PD0325901, PD0332991, PF02341066). The random forest algorithm was the most powerful approach. Using random forest, twelve of the fifteen signatures successfully predicted drug response with a precision greater than 0.80 (17-AAG, AZD0530, AZD6244, Erlotinib, Lapatinib, Nultin-3, Paclitaxel, PD0325901, PD0332991, PF02341066, PLX4720, Sorafenib).

We were unable to generate predictive signatures for three of the fifteen drugs of interest: Nilotinib, NVPTAE684, and PHA665752 (Figure 2). NVP-TAE684 and
Nilotinib target the protein products of gene fusions, $N P M-A L K$ and $B C R-A B L$ respectively. These gene fusions were not well represented in our datasets, making signature generation difficult. The low number of cell lines in the datasets sensitive to PHA665752 contributed to the difficulty of generating a predictive signature with good precision for this drug. While a signature could not be generated for PHA665752 reaching our precision cutoff of 0.80 , the random forest and support vector machine signatures, with precisions of 0.76 and 0.78 , greatly outperformed elastic net regression, which achieved a precision of 0.58 . The performance of the non-linear algorithms was markedly superior to that of the linear regression algorithm when $\mathrm{N}$, the number of cell lines sensitive to the drug of interest, was very small in comparison to $\mathrm{p}$, the total number of multi-omic features.

The predictive performance of the multi-omic signatures was tested against the CCLE and NCI60 datasets for robustness (Figure 3). Only 50\% of the signatures generated using elastic net regression and support vector machine could be validated on independent datasets. In comparison, $75 \%$ of the signatures generated using random forest were validated on independent datasets. Four out of the eight signatures developed using elastic net regression retained predictive precision greater than 0.80 when tested on the CCLE dataset (17-AAG, Lapatinib, PD0325901, PD0332991). Five out of the nine signatures developed using support vector machine retained predictive precision greater than 0.80 when tested on the CCLE dataset (17-AAG, AZD0530, Lapatinib, PD0332991, PF02341066). Random forest yielded more, and more robust predictive signatures, with nine out of the twelve signatures generated remaining predictive when tested against the CCLE dataset (17-AAG, AZD6244, Erlotinib, Lapatinib, Nultin-3, Paclitaxel, PD0325901, PD0332991, PLX4720). Response to the drug Sorafenib could not be independently validated using any of the generated signatures. Sorafenib is a multi-kinase inhibitor and it is likely that limiting our signatures to thirty features each did not allow enough genomic complexity to predict response to this drug.

\section{Random forest signatures}

The random forest algorithm identified high expression of NQO1 as the single most important and robust predictor of a cell's sensitivity to 17-AAG, an HSP90 inhibitor. Oncogenic proteins such as Raf- 1 and p53 are kept in an apoptosis resistant state by direct association with HSP90; heat shock protein inhibitors such as 17-AAG break the direct association between HSP90 and apoptotic proteins. NQO1 is a member of the $\mathrm{NAD}(\mathrm{P}) \mathrm{H}$ dehydrogenase quinone family and produces the highly potent and stable intermediate 17-AAGH2 when the compound 17-AAG is metabolized [24]. High expression of NQO1 has been 
previously reported in biological studies as a biomarker of sensitivity to $17-$ AAG $[5-7,25]$.

The predictive signature of response to PD-0325901 generated by random forest confirmed several previously identified biomarkers and identified several novel biomarkers. PD-0325901, a MEK inhibitor, directly affects MAPK signaling. Given the large number of genes involved in the $M A P K$ pathway, it is not surprising that the non-linear random forest approach best captured the many interacting genes that predict PD-0325901 response. The random forest approach confirmed previous knowledge indicating that high expression of SPRY2, LGALS3, and PHLDA1 predict sensitivity to PD-0325901: SPRY2 suppresses cell growth and differentiation by inhibiting the MAPK pathway [26]; LGALS3 modulates cell-cell and cell-matrix interactions [27]; and PHLDA1 is known to play an important role regulating anti-apoptotic effects [28]. The random forest approach confirmed this knowledge by highly weighting these genes in the predictive signature. Our study offers the novel finding that expression of GSN, PHF17, and ZFP30 is involved in conferring cellular sensitivity to PD-0325901: GSN is involved in the assembly and disassembly of actin filaments needed for cellular replication [29]; PHF17 is a known tumor suppressor and promotes apoptosis [30]; and ZFP30 is thought to be involved in regulating transcription.

The random forest algorithm was uniquely successful in generating predictive signatures for cytotoxic drugs, a result that was not achieved using linear regression[7]. In the case of the broadly acting taxane drug Paclitaxel, the random forest generated signature was precise and robust. Paclitaxel sensitivity is predicted by up regulation of and amplification in the genes ANXA1, SSRP1, PAFAH1B2, and PSMG3: ANXA1 inhibits the activation of NF-kB by binding to 655 [31]; SSRP1 forms the chromatin transcription elongation factor FACT which is crucial to the anticancer mechanism of DNA damaging drugs [32]; and PSMG3 is a proteasome assembly chaperone [33].

\section{A case study: triple negative breast cancer}

Triple negative breast cancer (TNBC) is defined by the absence of detectable estrogen and progesterone receptors and the lack of amplification in the human epidermal growth factor receptor 2 gene [34]. Triple negative breast cancer accounts for $15-20 \%$ of all breast cancers, is generally more aggressive, and patients have decreased overall survival [35]. TNBC does not represent a single type of breast cancer, but rather a heterogeneous group of tumors with distinct molecular subtypes. The triple negative subgroups respond differentially to drug therapy. The current standard of care for TNBC is treatment with taxanes and other cytotoxic compounds. While overall response to taxane treatment is $28 \%$, some TNBC groups such as the luminal androgen receptor subtype have a response to taxane drugs as low as $0-10 \%$ [34]. The primary clinical problem for TNBCs is the lack of targeted therapies and a standard by which to stratify patients into the available treatments. There are currently no triple negative breast cancer drugs in phase III clinical trials, highlighting the need to identify research, repositioned, and repurposed drug compounds to treat TNBC patients [36].

We applied our random forest drug response prediction signature, generated using Cancer Genome Project (CGP) data, to TNBC cell lines in the Cancer Cell Line Encyclopedia (CCLE). We predicted that $32 \%$ of the TNBC cell lines would be sensitive to treatment with Paclitaxel. Seven out of twenty-five (28\%) TNBC cell lines were true positives for sensitivity to treatment with the taxane drug Paclitaxel. This result is consistent with clinical results that indicate $28 \%$ of TNBC tumors respond to treatment with taxane drugs [34]. Additionally, we correctly predicted that a subset of triple negative breast cancer cell lines would be sensitive to treatment with 17-AAG. The group of TNBC cell lines with predicted and true sensitivity to 17-AAG belongs to the luminal androgen receptor subtype, a group that resists traditional treatment. As predicted there was a positive correlation between NQO1 expression and TNBC cellular sensitivity to 17 -AAG (Pearson correlation coefficient: 0.61). The random forest generated prediction signature also correctly predicted that $50 \%$ of triple negative breast cancer cell lines would be sensitive to the MEK inhibitor PD-0325901. The sensitive cell lines roughly correspond to the basal triple negative breast cancer subtype.

TNBC remains a challenging disease; here we have identified two promising research compounds for the treatment of TNBC. Preclinical identification of promising drug compounds, such as used in the approach described in this study, offer great promise to improve treatment of TNBC.

\section{Conclusions}

Using the random forest algorithm and support vector machine, we were able to generate and validate robust multi-omic signatures that predict drug response to 17 AAG, AZD0530, AZD6244, Erlotinib, Lapatinib, Nultin-3, Paclitaxel, PD0325901, PD0332991, PF02341066, and PLX4720. The non-linear machine learning techniques random forest and support vector machine outperformed the more commonly used elastic net regression in developing precise and robust genomic predictors. Our results suggest that large pharmacogenomic databases can be used to identify the genomic correlates of anticancer drug response. The resulting classification of multi-omic predictors of drug response could be used to stratify patients into treatment groups based on their individual tumor biology. Prediction signatures show special promise for diseases, such as triple negative breast cancer, where there 
remains an urgent need to identify research or repositioned compounds that can be developed as targeted treatment for this difficult to treat patient population.

Our research could be extended in multiple ways: lineage specific predictors of drug response could be explored; further tuning of machine learning parameters could yield improved prediction results; and signature including features of the epigenome and proteome could improve drug response prediction.

\section{Competing interests}

The authors declare that they have no competing interests.

\section{Authors' contributions}

LCS designed the experiment, performed the bioinformatics analysis, and drafted the manuscript. TP assisted with bioinformatics analysis. YC assisted in data generation. JBS conceived of the study and revised the manuscript.

\section{Acknowledgements}

We would like to thank Mehmet Koyuturk, Afshin Dowlati, and Gary Wildey for helpful discussions and Kaide Zhai for contributing to background research. This work is funded by a National Science Foundation Graduate Research Fellowship (Grant No. DGEO 9517830) and Case Comprehensive Cancer Center Support (Grant No. P30 CA043703).

This article has been published as part of BMC Genomics Volume 15 Supplement 7, 2014: Proceedings of the 9th International Conference of the Brazilian Association for Bioinformatics and Computational Biology (XMeeting 2013). The full contents of the supplement are available online at http://www.biomedcentral.com/bmcgenomics/supplements/15/S7.

\section{Authors' details}

${ }^{1}$ Case Comprehensive Cancer Center. ${ }^{2}$ Center for Proteomics and Bioinformatics, Case Western Reserve University, Cleveland, Ohio 44106 USA. ${ }^{3}$ Department of Biological Engineering, Massachusetts Institute of Technology, Cambridge, Massachusetts 02139 USA

Published: 27 October 2014

\section{References}

1. Shoemaker $\mathrm{RH}$ : The $\mathrm{NCl} 60$ human tumour cell line anticancer drug screen. Nature reviews Cancer 2006, 6(10):813-823.

2. Chapman PB, Hauschild A, Robert C, Haanen JB, Ascierto P, Larkin J, Dummer R, Garbe C, Testori A, Maio M, et al: Improved survival with vemurafenib in melanoma with BRAF V600E mutation. The New England journal of medicine 2011, 364(26):2507-2516.

3. Kwak EL, Bang YJ, Camidge DR, Shaw AT, Solomon B, Maki RG, Ou SH, Dezube BJ, Janne PA, Costa DB, et al: Anaplastic lymphoma kinase inhibition in non-small-cell lung cancer. The New England journal of medicine 2010, 363(18):1693-1703.

4. Druker BJ, Guilhot F, O'Brien SG, Gathmann I, Kantarjian H, Gattermann N, Deininger MW, Silver RT, Goldman JM, Stone RM, et al: Five-year follow-up of patients receiving imatinib for chronic myeloid leukemia. The New England journal of medicine 2006, 355(23):2408-2417.

5. Barretina J, Caponigro G, Stransky N, Venkatesan K, Margolin AA, Kim S, Wilson CJ, Lehar J, Kryukov GV, Sonkin D, et al: The Cancer Cell Line Encyclopedia enables predictive modelling of anticancer drug sensitivity. Nature 2012, 483(7391):603-607

6. Garnett MJ, Edelman EJ, Heidorn SJ, Greenman CD, Dastur A, Lau KW, Greninger P, Thompson IR, Luo X, Soares J, et al: Systematic identification of genomic markers of drug sensitivity in cancer cells. Nature 2012, 483(7391):570-575

7. Papillon-Cavanagh S, De Jay N, Hachem N, Olsen C, Bontempi G, Aerts HJ, Quackenbush J, Haibe-Kains B: Comparison and validation of genomic predictors for anticancer drug sensitivity. Journal of the American Medical Informatics Association: JAMIA 2013, 20(4):597-602.

8. McCall MN, Bolstad BM, Irizarry RA: Frozen robust multiarray analysis (fRMA). Biostatistics 2010, 11(2):242-253.
9. Durinck S, Moreau Y, Kasprzyk A, Davis S, De Moor B, Brazma A, Huber W: BioMart and Bioconductor: a powerful link between biological databases and microarray data analysis. Bioinformatics 2005, 21(16):3439-3440.

10. Leek JT, Storey JD: Capturing heterogeneity in gene expression studies by surrogate variable analysis. PLoS genetics 2007, 3(9):1724-1735.

11. Li Q, Birkbak NJ, Gyorffy B, Szallasi Z, Eklund AC: Jetset: selecting the optimal microarray probe set to represent a gene. BMC bioinformatics 2011, 12:474.

12. Greenman CD, Bignell G, Butler A, Edkins S, Hinton J, Beare D, Swamy S, Santarius T, Chen L, Widaa S, et al: PICNIC: an algorithm to predict absolute allelic copy number variation with microarray cancer data. Biostatistics 2010, 11(1):164-175.

13. Dudoit S, Fridlyand J, Speed TP: Comparison of discrimination methods for the classification of tumors using gene expression data. J Am Stat Assoc 2002, 97(457):77-87.

14. Haibe-Kains B, Desmedt C, Sotiriou C, Bontempi G: A comparative study of survival models for breast cancer prognostication based on microarray data: does a single gene beat them all? Bioinformatics 2008, 24(19):2200-2208.

15. Breiman L: Random Forests. Machine Learning 2001, 45(1):5-32.

16. Ishwaran $\mathrm{H}$, Kogalur UB, Blackstone EH, Lauer MS: Random Survival Forests. Ann Appl Stat 2008, 2(3):841-860.

17. Dimitriadou E, Hornik K, Leisch F, Meyer D, Weingessel A: e1071: Misc Functions of the Department of Statistics(e1071). TU Wien 2011

18. Friedman J, Hastie T, Tibshirani R: Regularization paths for generalized linear models via coordinate descent. Journal of statistical software 2010, 33(1):1.

19. Zhang W, Ota T, Shridhar V, Chien J, Wu B, Kuang R: Network-based survival analysis reveals subnetwork signatures for predicting outcomes of ovarian cancer treatment. PLoS computational biology 2013, 9(3): e1002975.

20. Brown MP, Grundy WN, Lin D, Cristianini N, Sugnet CW, Furey TS, Ares M, Haussler D: Knowledge-based analysis of microarray gene expression data by using support vector machines. Proceedings of the National Academy of Sciences 2000, 97(1):262-267.

21. Furey TS, Cristianini N, Duffy N, Bednarski DW, Schummer M, Haussler D: Support vector machine classification and validation of cancer tissue samples using microarray expression data. Bioinformatics 2000, 16(10):906-914.

22. Hua S, Sun Z: Support vector machine approach for protein subcellular localization prediction. Bioinformatics 2001, 17(8):721-728.

23. Noble WS: Support vector machine applications in computational biology. Kernel methods in computational biology 2004, 71-92.

24. Guo W, Reigan P, Siegel D, Zirrolli J, Gustafson D, Ross D: Formation of 17-allylamino-demethoxygeldanamycin (17-AAG) hydroquinone by NAD (P)H:quinone oxidoreductase 1: role of 17-AAG hydroquinone in heat shock protein 90 inhibition. Cancer research 2005, 65(21):10006-10015.

25. Kelland LR, Sharp SY, Rogers PM, Myers TG, Workman P: DT-Diaphorase expression and tumor cell sensitivity to 17-allylamino, 17demethoxygeldanamycin, an inhibitor of heat shock protein 90 . Journal of the National Cancer Institute 1999, 91(22):1940-1949.

26. Wong KK: Recent developments in anti-cancer agents targeting the Ras/ Raf/ MEK/ERK pathway. Recent patents on anti-cancer drug discovery 2009, 4(1):28-35.

27. Cheng CL, Hou HA, Lee MC, Liu CY, Jhuang JY, Lai YJ, Lin CW, Chen HY, Liu FT, Chou WC, et al: Higher bone marrow LGALS3 expression is an independent unfavorable prognostic factor for overall survival in patients with acute myeloid leukemia. Blood 2013, 121(16):3172-3180.

28. Nagai MA, Fregnani JH, Netto MM, Brentani MM, Soares FA: Downregulation of PHLDA1 gene expression is associated with breast cancer progression. Breast cancer research and treatment 2007, 106(1):49-56.

29. Baig RM, Mahjabeen I, Sabir M, Masood N, Ali K, Malik FA, Kayani MA: Mutational spectrum of Gelsolin and its down regulation is associated with breast cancer. Disease markers 2013, 34(2):71-80.

30. Zhou MI, Foy RL, Chitalia VC, Zhao J, Panchenko MV, Wang H, Cohen HT: Jade-1, a candidate renal tumor suppressor that promotes apoptosis. Proceedings of the National Academy of Sciences of the United States of America 2005, 102(31):11035-11040.

31. Huo XF, Zhang JW: Annexin1 regulates the erythroid differentiation through ERK signaling pathway. Biochemical and biophysical research communications 2005, 331(4):1346-1352. 
32. Zeng SX, Dai MS, Keller DM, Lu H: SSRP1 functions as a co-activator of the transcriptional activator p63. The EMBO journal 2002, 21(20):5487-5497.

33. Roelofs J, Park S, Haas W, Tian G, McAllister FE, Huo Y, Lee BH, Zhang F,

Shi Y, Gygi SP, et al: Chaperone-mediated pathway of proteasome regulatory particle assembly. Nature 2009, 459(7248):861-865.

34. Lehmann BD, Pietenpol JA: Identification and use of biomarkers in treatment strategies for triple-negative breast cancer subtypes. The Journal of pathology 2014, 232(2):142-150.

35. Ferguson LL, Curran B, Martinez M, Mancuso P: Triple-negative breast cancer: what is known about it? Clinical journal of oncology nursing 2014 18(1):E6-E11.

36. Turner NC, Reis-Filho JS: Tackling the diversity of triple-negative breast cancer. Clinical cancer research : an official journal of the American Association for Cancer Research 2013, 19(23):6380-6388.

doi:10.1186/1471-2164-15-S7-S2

Cite this article as: Stetson et al: Computational identification of multiomic correlates of anticancer therapeutic response. BMC Genomics 2014 15(Suppl 7):S2.

\section{Submit your next manuscript to BioMed Central} and take full advantage of:

- Convenient online submission

- Thorough peer review

- No space constraints or color figure charges

- Immediate publication on acceptance

- Inclusion in PubMed, CAS, Scopus and Google Scholar

- Research which is freely available for redistribution

Submit your manuscript at www.biomedcentral.com/submit
C Biomed Central 